\title{
Chemical analysis of acoustically levitated drops by Raman spectroscopy
}

\author{
Rudolf Tuckermann • Ljiljana Puskar • \\ Mahta Zavabeti • Ryo Sekine • Don McNaughton
}

Received: 20 November 2008 /Revised: 9 April 2009 /Accepted: 9 April 2009/Published online: 6 May 2009

(C) Springer-Verlag 2009

\begin{abstract}
An experimental apparatus combining Raman spectroscopy with acoustic levitation, Raman acoustic levitation spectroscopy (RALS), is investigated in the field of physical and chemical analytics. Whereas acoustic levitation enables the contactless handling of microsized samples, Raman spectroscopy offers the advantage of a noninvasive method without complex sample preparation. After carrying out some systematic tests to probe the sensitivity of the technique to drop size, shape, and position, RALS has been successfully applied in monitoring sample dilution and preconcentration, evaporation, crystallization, an acid-base reaction, and analytes in a surface-enhanced Raman spectroscopy colloidal suspension.
\end{abstract}

Keywords Raman spectroscopy · Acoustic levitation · Drop evaporation $\cdot$ Crystallization $\cdot$ Microtitration

\footnotetext{
R. Tuckermann

Institute of Physical Chemistry, University of Göttingen,

Tamannstr. 6,

37077 Göttingen, Germany

L. Puskar $\cdot$ M. Zavabeti $\cdot$ R. Sekine $\cdot$ D. McNaughton $(\square)$

Center for Biospectroscopy and School of Chemistry,

Monash University,

Wellington Rd,

Clayton, Victoria 3800, Australia

e-mail: don.mcnaughton@sci.monash.edu.au

L. Puskar

Australian Synchrotron,

800 Blackburn Rd,

Clayton, Victoria 3168, Australia
}

\section{Introduction}

Small sample volumes generate many of today's analytical problems that can only be solved through a corresponding miniaturization of the analytical instrumentation. For this reason, microtechnology and nanotechnology have been introduced to the field of analytical chemistry. While analytical methods based on spectroscopic techniques are sufficiently sensitive for the analysis of trace elements, the handling of small sample volumes in the submicroliter range is inherently difficult. As the sample volumes become smaller, increased surface-to-volume ratios result in a higher chance of contamination, sorption, and other heterogeneous processes through the container walls.

Acoustic levitation has been demonstrated by Lierke [1] to be a useful technique for contactless handling of small solid and liquid samples in a gaseous environment. Sample sizes are typically in the range of 5-5,000 $\mathrm{nL}$ corresponding to diameters of $0.2-2 \mathrm{~mm}$, respectively. Because of the lack of containment, acoustic levitation inhibits wall effects and offers a contactless handling medium for microsized samples. Welter and Neidhart [2] were the first to introduce acoustic levitation to analytical chemistry and recently many benefits and advantages of this technique in analytical chemistry have been emphasized in two review articles by Santesson and Nilsson [3] and Priego-Capote and Luque de Castro [4].

Raman spectroscopy as a noninvasive and water- and air-insensitive method with no requirement for complex sample preparation has also become a very attractive tool for analyzing microscopic samples [5]. It offers good sensitivity and selectivity to changes of the physicochemical properties of many organic and inorganic substances on 
a molecular scale as well as a time resolution of a few seconds for monitoring online processes.

The combination of Raman spectroscopy with acoustic levitation, Raman acoustic levitation spectroscopy (RALS), enables a time-resolved contactless monitoring of in situ chemical reactions and physical and biological processes in a single drop (or particle) suspended in a gaseous environment. The acoustic levitation technique, first coupled to Raman spectroscopy by Biswas $[6,7]$ to follow the solidification of acoustically levitated crystals, has since been improved and adapted by several other groups: while Davies et al. have used acoustic levitation for simplified microsample handling in laser spectroscopy [8], Raman spectroscopy has been used for online monitoring of airborne chemistry [9] and of protein crystallization [10] in acoustically levitated drops. Furthermore, Raman spectroscopy on deformed levitated drops has been experimentally and theoretically investigated by Esen et al. [11]. The group of Lendl has successfully coupled acoustic field levitation with flow through methods in samples such as ionic liquids [12] and surface-enhanced Raman spectroscopy (SERS) in silver sols $[9,13]$. The application of the RALS technique to the field of biospectroscopy has recently been applied by Puskar et al. in monitoring the malarial pigment hemozoin in live malarial-infected levitated red blood cells [14] and also for online monitoring of environmentally stressed live algae by Wood et al. [15].

While in most of the investigations mentioned above RALS has been used for qualitative monitoring, the major purpose of the present work has been to probe the utilization of RALS in quantitative analytical chemistry. Therefore, the technique has been investigated in the simple chemical and physicochemical processes of preconcentration, evaporation, an acid-base reaction, crystallization, and with SERS solutions to determine quantification limits.

\section{Material and method}

\section{Chemicals}

All chemicals used in this investigation were purchased from commercial sources.

\section{Dipicolinic acid solutions}

A $10^{-3}-\mathrm{M}$ stock solution was initially prepared by dissolving $0.0167 \mathrm{~g}$ of dipicolinic acid (DPA) powder into $100 \mathrm{ml}$ of $18.2-\mathrm{M} \Omega \mathrm{MQ}$ distilled water. The stock solution was diluted into lower concentrations including $5 \times 10^{-4}, 1 \times 10^{-4}$, and $5 \times 10^{-5} \mathrm{M}$.

\section{Silver colloid preparation}

Silver colloid was prepared by the method of Lee and Meisel [16].

Briefly, $90 \mathrm{mg}$ of $\mathrm{AgNO}_{3}$ was dissolved in $500 \mathrm{ml}$ of 18.2-M $\Omega$ MQ distilled water and brought to boiling. A solution of $1 \%$ sodium citrate $(10 \mathrm{~mL})$ was added and the solution was kept boiling for $1 \mathrm{~h}$. The Ag colloid solution thus prepared was greenish yellow and had an absorption maximum at $408 \mathrm{~nm}$.

\section{Solution for SERS preparation}

Two hundred microliters of DPA solutions were added to $100 \mu \mathrm{L}$ of $\mathrm{Ag}$ colloid followed by $35 \mu \mathrm{L}$ of $0.1 \mathrm{M} \mathrm{Na}_{2} \mathrm{SO}_{4}$ as aggregation agent.

\section{Experimental apparatus}

A schematic drawing of the experimental setup is shown in Fig. 1. It consists of an acoustic levitator (Dantec/Invent Measurements Technology, Erlangen, Germany) coupled to a commercial Renishaw confocal micro-Raman system 2000 spectrometer with a Peltier-cooled charge-coupled device (CCD) detector. The acoustic levitator and the principles of acoustic levitation are described in detail by Lierke [1] so only a brief account is given here. It has been found that in the pressure nodes of a strong stationary ultrasonic field small liquid and solid samples can be suspended in a gaseous environment at sound pressure levels of about $165 \mathrm{~dB}$. The ultrasound frequency of the one-axial acoustic levitator used in this investigation was $58 \mathrm{kHz}$, while typical start volumes of the drops ranged between 0.5 and $5 \mu \mathrm{L}$ corresponding to diameters of the levitated drops between about 1.0 and $2.0 \mathrm{~mm}$. The drops were created by a microliter syringe and were directly inserted into the central pressure node of the stationary ultrasonic field.

In order to use the acoustic levitator in a vertical alignment and take advantage of the stronger axial (than radial) levitation forces, the excitation laser beam emerging from the Raman spectrometer and down through a modified BH2-UMA Olympus optical microscope was diverted with a $90^{\circ}$ adaptor (Wotton Scientific) holding a $16-\mathrm{mm}$ focal length lens. The acoustic levitator was mounted on an $x y z$ micropositioning stage for alignment of the sample and a visible microscope. The laser source was a 782-nm diode laser with a maximum power of $25 \mathrm{~mW}$ or a $633-\mathrm{nm} \mathrm{He} / \mathrm{Ne}$ laser with a maximum power of $32 \mathrm{~mW}$. Power at the sample was typically 2 to $3 \mathrm{~mW}$. The scattered light is collected along the same optical path as the incoming laser ( $180^{\circ}$ geometry). Raman-scattered light separated from the Rayleigh and anti-Stokes light component by an edge cutoff 
Fig. 1 Schematic drawing of the experimental apparatus

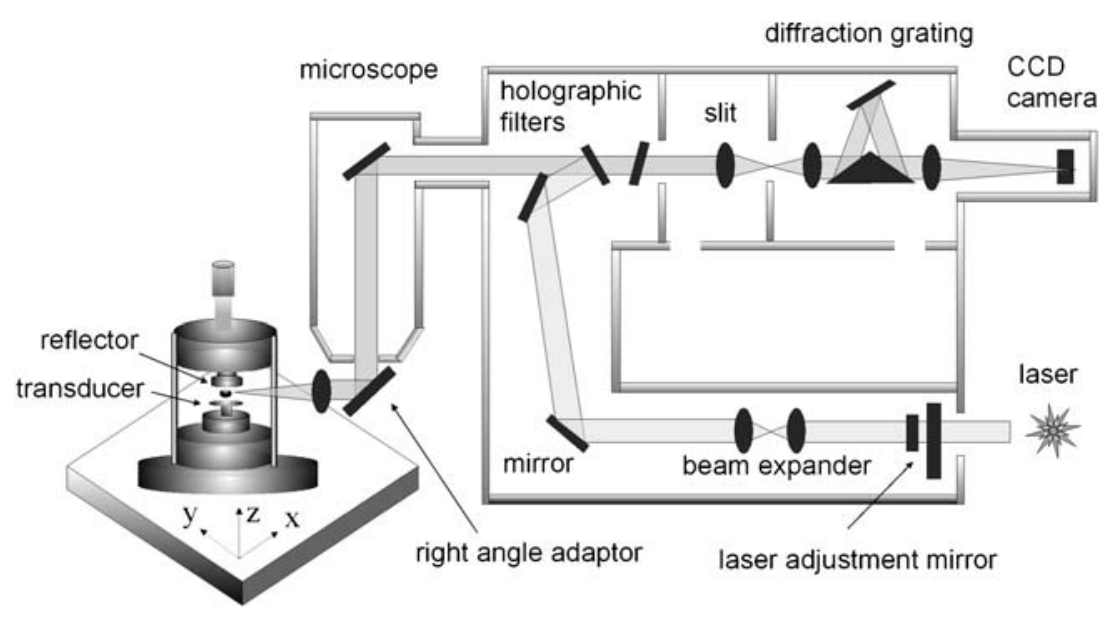

filter is focused onto the slit assembly, recollimated and reflected onto a diffraction grating that disperses the scattered light over a Peltier-cooled CCD array detector. The spectrometer is controlled by a PC using two software packages, instrument control software (Renishaw WiRETM) and data analysis software (Galactic GRAMS/32 ${ }^{\mathrm{TM}}$ ). All spectra were collected with 5-30 s of laser exposure in the region between 1,800 and $200 \mathrm{~cm}^{-1}$ and at a spectral resolution of approximately $2 \mathrm{~cm}^{-1}$.

Systematic tests of RALS technique

Systematic tests were carried out to probe the sensitivity of the RALS technique to drop size, shape, and its distance to the objective lens of the microscope. The droplet shape is probably more accurately described by the drop deformation, i.e., the ratio of horizontal to vertical drop diameter, and can be determined, e.g., from backlit images taken with an intelligent CCD camera [17]. The drop deformation is primarily a function of the sound pressure level of the stationary ultrasonic field: higher sound pressure levels instigate the flattening of the levitated drop with it becoming a more oblate rotational ellipsoid with respect to the vertical levitation axis. Esen et al. [11] observed an increase in the Raman intensity of approximately $50 \%$ for the deformed nonspherical droplet when compared to a spherical one of the same volume. But this effect was measured with an illuminating laser beam approximately ten times larger than the drop diameter and the light collecting optics at $90^{\circ}$ angle with respect to the incoming laser. In our setup, the intensity of the scattered light was measured at $180^{\circ}$ and the laser spot on the levitated droplet was approximately the same as a typical drop diameter.
As shown in Fig. 2, in the case of levitated dimethyl sulfoxide (DMSO) drops, we have measured the intensity of the very strong Raman band at $669 \mathrm{~cm}^{-1}$ by changing the $x y z$ position of the drop with respect to the focal point. The $x$ direction was defined in the direction of the incoming laser beam, the $z$ direction in the direction of the (vertical) levitation axis, and the $y$ direction perpendicular to both. By changing the $z$ position of the levitated drop, two maxima are observable when the focal point reaches the drop surface (Fig. 2c). In principle, the same effect should be also observed in the $y$ direction. But, as the levitation forces are stronger in the axial $(z)$ than in the radial $(x y)$ direction, levitated drops are slightly oscillating around the pressure node more in the $x y$ plane than in the $z$ direction. Therefore, the obtained intensity profiles are more smoothed in the $y$ direction than in $z$ direction, losing the maxima at the drop surface. In the $x$ direction, scattering effects of the incoming laser beam have to be taken into account, resulting in an asymmetric intensity profile, e.g., if the focal point of the incoming laser beam is behind the levitated drop, more light is backscattered than when the focal point is in front of the levitated drop. In all the following experiments, the focal point of the incoming laser beam was adjusted to be in the middle of the levitated drop. Besides these effects, the signal seems to be independent of drop size and shape.

One of the advantages of acoustic levitation is the contactless handling of microsamples. But the effectiveness of collecting backscattered light from an acoustically levitated microliter drop in our system is lower than from liquid bulk phase in the milliliter range contained in a small glass vessel and positioned in the focal point of the same micro-Raman system. As shown in Fig. 3 for ethanol, band intensities decrease by a factor of about 2 , which 


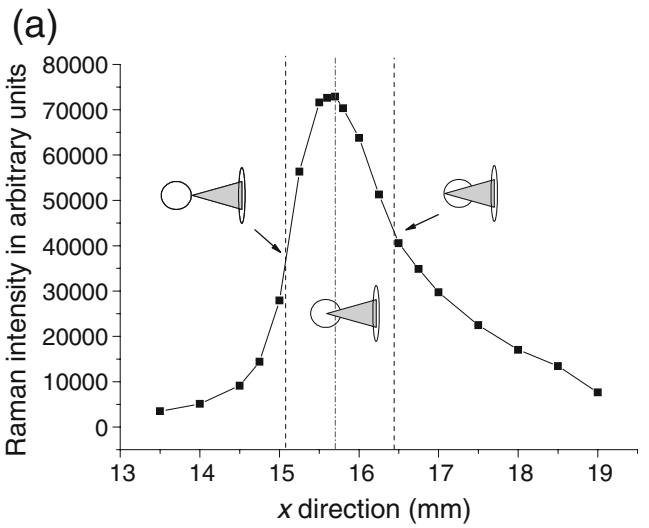

(b)

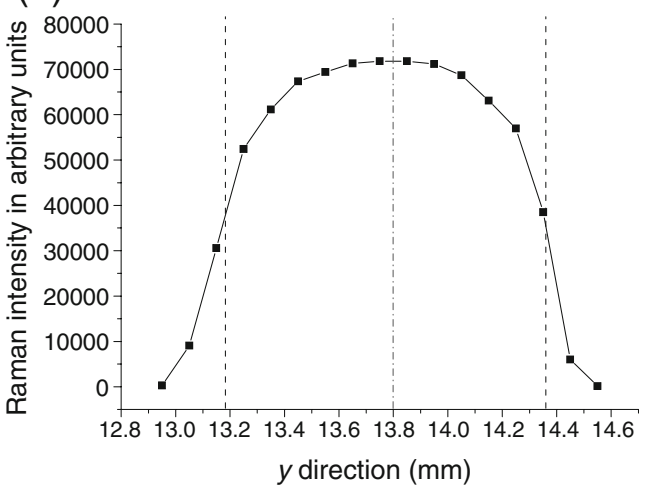

(c)

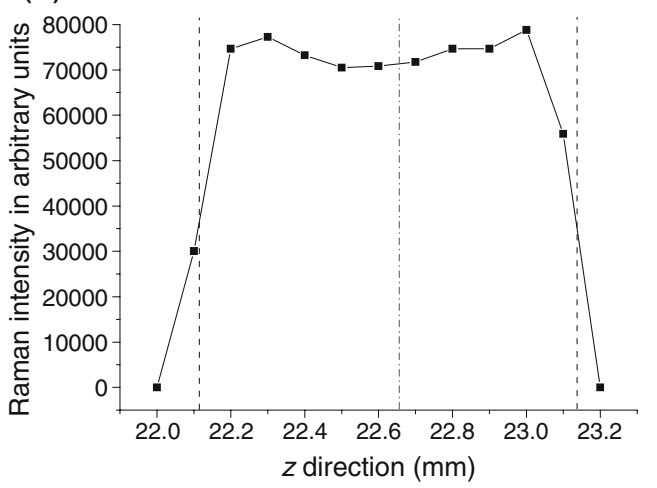

Fig. 2 Intensity profiles of an acoustically levitated DMSO drop of approximately $1 \mu \mathrm{L}$ measured for the very strong Raman band at $669 \mathrm{~cm}^{-1}$ by changing the $x y z$ position of the drop with respect to the focal point. The outer dashed lines in each graph determine the border of the drop, while the inner dashed line indicates the $x y z$ position which was normally used. In a, the focal point of the incoming laser beam was in front of the levitated drop on the left side of the graph while it was behind the drop on the right side of the graph. For all plots, the laser was positioned at the central position of the other two axes

may be due to a greater interaction volume in a bulk sample because the laser interaction depth is typically greater than the diameter of the laser spot. This disadvantage of the RALS technique may possibly be improved by using higher aperture optics minimizing the distance between acoustically levitated drop and the collection

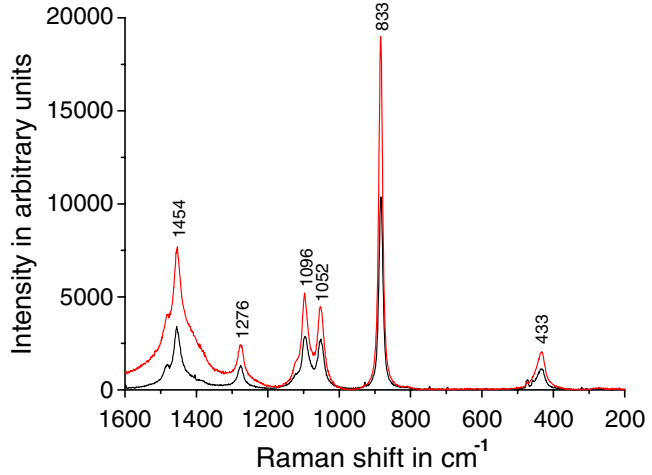

Fig. 3 Raman spectra (sampling time of 30 s) of ethanol in an acoustically levitated drop (black) and glass vessel (red). Band positions and relative intensities are in good agreement with the reference data of Schrader [29]

lens. The signal-to-noise ratio of strong normal Raman bands was in the range of 100 to 1,000 .

\section{Results and discussion}

Concentration series of DMSO in an acoustically levitated water drops

Preconcentration and determination of analytes in acoustically levitated drops using visible absorption spectroscopy and fluorescence spectroscopy have been carried out by Rohling et al. [18]. Both are required for quantitative analysis of chemical compounds in acoustically levitated drops. In order to demonstrate the use of the RALS technique in quantitative analytical chemistry, a concentration series of a dissolved chemical compound, DMSO, in a suspended water drop was measured (see Fig. 4).

In quantitative analytical chemistry, the detection limit is usually defined as a signal-to-noise ratio of $3(S / N=3)$. Applied to the very strong symmetric $\mathrm{C}-\mathrm{S}$ stretching mode of DMSO at $669 \mathrm{~cm}^{-1}$, for which we have measured a signal-to-noise ratio of approximately 1,200 in this work, the detection limit of DMSO ranges between $2 \%$ and $3 \%$ for the volume mixing ratio of DMSO in water. This result demonstrates that conventional Raman spectroscopy, although suitable for quantification of analytes in relatively high concentration is not very suitable in quantitative analytical chemistry for low concentrations, although the detection of target compounds is possible without any sample preparation or the use of indicators.

However, conventional Raman spectroscopy is very responsive to changes in the physicochemical properties of the molecular structure such as length and coupling strength of molecular bonds. Therefore, the vibrational modes of molecular bonds are shifted due to changes in the 
Fig. 4 Raman spectra (sampling time $30 \mathrm{~s}$ ) of acoustically levitated drops (approximately $5 \mu \mathrm{L}$ ) containing DMSO and water. a Shows the spectral region of the symmetric and antisymmetric $\mathrm{C}-\mathrm{S}$ stretching modes; $\mathbf{b}$ the symmetric $\mathrm{S}=\mathrm{O}$ stretching mode and the methyl rocking mode; $\mathbf{c}$ highlights the evolution of band intensity and band position of the very strong symmetric $\mathrm{C}-\mathrm{S}$ stretching mode at $672 \mathrm{~cm}^{-1}$. (Note: in contrast to reference data of Schrader [29], the band positions of the symmetric and antisymmetric $\mathrm{C}-\mathrm{S}$ vibration modes of pure DMSO are slightly blue-shifted while the band position of the symmetric $\mathrm{S}=\mathrm{O}$ stretching mode is slightly red-shifted. Both are probably caused by a small amount of water in the DMSO samples) (a)

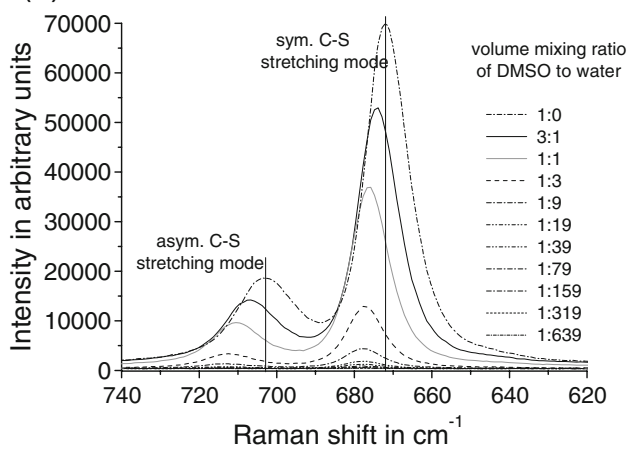

(b)

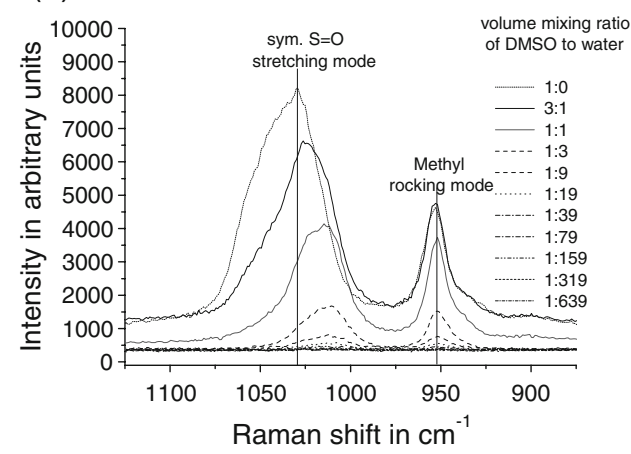

(c)

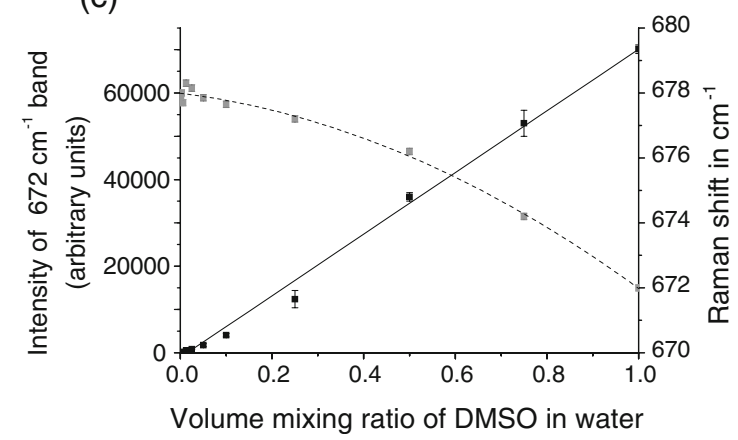

Volume mixing ratio of DMSO in water

molecular environment (e.g., phase transition, crystallization, and solvent effects). The dilution of DMSO with water is an example of its usefulness for monitoring physicochemical changes: as shown in Fig. 4, the symmetric $\left(670 \mathrm{~cm}^{-1}\right)$ and antisymmetric $\left(699 \mathrm{~cm}^{-1}\right) \mathrm{C}-\mathrm{S}$ stretching modes are blue-shifted due to an increase in water present around the DMSO molecules. In contrast to that, a red shift for the symmetric $\mathrm{S}=\mathrm{O}$ stretching mode at $1,029 \mathrm{~cm}^{-1}$ is observed while no shift is noted for the 1,419 - and $952-\mathrm{cm}^{-1}$ bands attributed to the methyl bending and symmetric methyl rocking vibrations. A blue shift of $\mathrm{C}-\mathrm{S}$ stretching modes and a red shift of the symmetric $\mathrm{S}=\mathrm{O}$ stretching mode were also reported in Raman spectra of DMSO in DMSOintercalated kaolinites [19]. Whereas the red shift of the $S=O$ stretching mode indicates a weakening of the $\mathrm{S}=\mathrm{O}$ bond, the blue shift of the $\mathrm{C}-\mathrm{S}$ stretching modes indicates a strengthening of the $\mathrm{C}-\mathrm{S}$ bond. Both effects are caused by hydrogen bonding between water and DMSO molecules. The lack of spectral shift for the $\mathrm{C}-\mathrm{H}$ modes confirms that the methyl groups do not take part in hydrogen bonding.

Surface-enhanced Raman spectroscopy of dipicolinic acid

The sensitivity of Raman spectroscopy can be dramatically improved via SERS by a factor of $10^{8}$ to $10^{12}[20,21]$, enabling even single-molecule detection. The SERS technique has been applied to acoustically levitated drops by Santesson et al. [10] and the group of Lendl [9, 12], enhancing the RALS technique by a factor of about $10^{5}$. In order to test our RALS setup with an analyte of significant importance, we have used DPA. DPA is present in the spores of Bacillus anthracis and SERS techniques are under development for rapid anthrax endospore detection [22].

Initial RALS experiments using the 782-nm laser gave the DPA spectrum in Fig. 5, and higher S/N spectra were achieved by using a 633-nm laser to better match the optimum frequency required for the aggregation condition of the colloid. By comparison, a levitated drop of pure

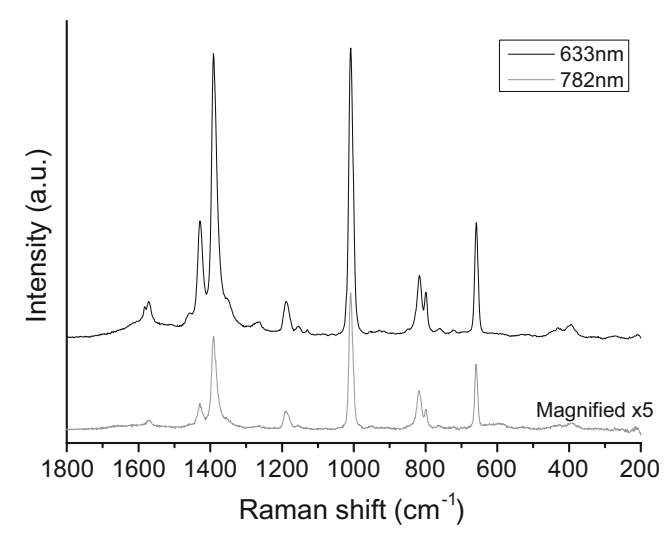

Fig. 5 Raman spectra (sampling time $10 \mathrm{~s}$ ) of acoustically levitated drops (approximately $5 \mu \mathrm{L}$ ) of SERS solutions of dipicolinic acid $\left(10^{-3} \mathrm{M}\right)$ using both 633-nm (top) and 782-nm lasers (bottom) 
$10^{-3}$-M DPA solution produced a spectrum where only bands due to water were observed. In Fig. 6, the RALS spectrum of successively more dilute solutions of DPA down to $5 \times 10^{-5} \mathrm{M}$ are presented showing that detection down to very low concentration is possible with the RALS/SERS combination. Injection of new droplets into the levitator gave spectra that were reproducible with respect to line intensities to within a few percent although, as also reported by Lendl [13], the signal strength decayed with time as the solution evaporated and the drop size changed. Injection of microdroplets of the SERS solution to maintain the volume resulted in the signals returning to their initial intensity.

Evaporation of acoustically levitated drops

The evaporation of acoustically levitated drops has been extensively investigated by Yarin et al. [23] and Tuckermann et al. [24] using the physical techniques of drop size measurement and temperature change. Evaporation of volatile solvents, e.g., alcohols with a short carbon chain, in a suspended drop at ambient conditions in humid air leads to condensation of water on the cold drop surface. As a pure solvent drop evolves via a mixed solvent-water drop to a pure water drop, changes in drop size, drop surface area, and drop temperature are observed.

An example of the evaporation of a levitated methanol drop in ambient air of $T=21{ }^{\circ} \mathrm{C}$ and $\mathrm{RH}=40 \%$ followed over time by Raman spectroscopy is given in Fig. 7. During the evaporation of levitated drops of such hydrophilic and volatile solvents, the mixing ratio of solvent and water changes and with it also the wavenumber and intensity of solvent Raman bands. Changes in drop size and drop surface temperature were also measured by a CCD camera and an infrared (IR) thermography system, respectively, in

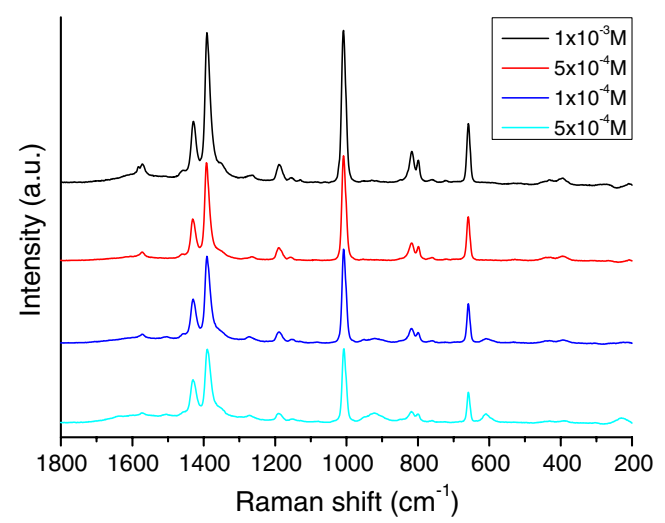

Fig. 6 Raman spectra (sampling time $10 \mathrm{~s}$ ) of acoustically levitated drops (approximately $5 \mu \mathrm{L}$ ) of SERS solutions of dipicolinic acid at different analyte concentrations using 633-nm laser the manner described in detail by Tuckermann et al. [24]. As water condenses out on the cold surface of the evaporating methanol drop, the drop temperature increases while drop surface area and intensity of the $\mathrm{C}-\mathrm{O}$ vibration band at $1,033 \mathrm{~cm}^{-1}$ decrease with time. Furthermore, the position of the Raman band is strongly red-shifted by approximately $17 \mathrm{~cm}^{-1}$ due to changes in the molecular environment of methanol: the hydration weakens the $\mathrm{C}-\mathrm{O}$ bond of methanol. (For comparison, in the case of evaporating ethanol drops, the $\mathrm{C}-\mathrm{O}$ vibration mode is only shifted from 882 to $879 \mathrm{~cm}^{-1}$ ). While the band intensity relating to the methanol-water mixing ratio decreases exponentially, the band shift declines approximately linearly during the time of evaporation in which the Raman band of methanol is detectable.

\section{Crystallization in acoustically levitated drop}

Evaporation of salt/water drops at ambient conditions results in saturated or oversaturated aqueous solution, from which crystallization can occur. Hydroscopic growth, including drop evaporation and crystallization (efflorescence) as well as condensation and deliquescence, has been reported using electrodynamically balanced water/salt microdroplets with Raman spectroscopy as a detection technique [25]. The crystallization results in characteristic shifts of Raman bands of the crystallizing salt.

The crystallization and evaporation of acoustically levitated salt/water drops have been exemplarily investigated on aqueous solution of ammonium sulfate $\left(\mathrm{NH}_{4}\right)_{2} \mathrm{SO}_{4}$ (see Fig. 8) and $\mathrm{Na}_{2} \mathrm{SO}_{4}$ (Fig. 9). The measurements in Figs. 8a and 9a were taken with a CCD camera and IR thermography system as described by Tuckermann et al. [24].

For a levitated drop of saturated aqueous solution of $\left(\mathrm{NH}_{4}\right)_{2} \mathrm{SO}_{4}$ at ambient room conditions, the crystallization started from inside the drop as observed by CCD camera. Crystalline edges appeared at the drop surface after approximately $5 \mathrm{~min}$ while most of the solution had crystallized after approximately 15 min resulting in an increase of temperature of the levitated drop/particle. The same process was followed by Raman spectroscopy which shows the $\mathrm{SO}_{4}{ }^{2-}$ band at $982 \mathrm{~cm}^{-1}$ (full width at half high $(\mathrm{FWHH})=9.7 \mathrm{~cm}^{-1}$ ) of aqueous solution red-shifting to $976 \mathrm{~cm}^{-1}\left(\mathrm{FWHH}=6.9 \mathrm{~cm}^{-1}\right)$ in the crystalline phase. These values are in good agreement with Raman results using optical trapping techniques to trap drops [26] and indicate a weakening of the $\mathrm{S}-\mathrm{O}$ bond during crystallization. The molecular transition of ammonium sulfate from aqueous solution to crystalline state mostly takes place after 8 to $9 \mathrm{~min}$, which cannot be observed in the macroscopic values of drop surface and drop temperature. Raman spectra recorded between 5 and 14 min show broadened bands 
Fig. 7 Evaporation of an acoustically levitated methanol drop in ambient air. a Drop surface and drop temperature were measured nearly each second using a CCD camera and an IR thermography system, respectively [24]. b Raman spectra were recorded nearly every minute with a sampling time of 5 s. c While intensity of the Raman band (black) is decreasing exponentially, its position (gray) is (red-)shifted linearly with time during drop evaporation (a)

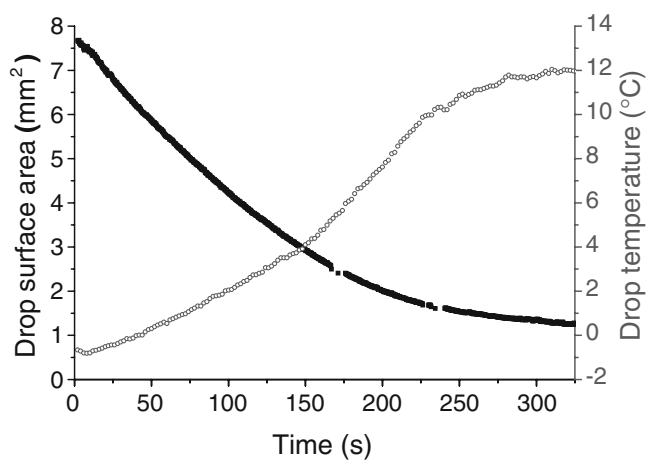

(b)

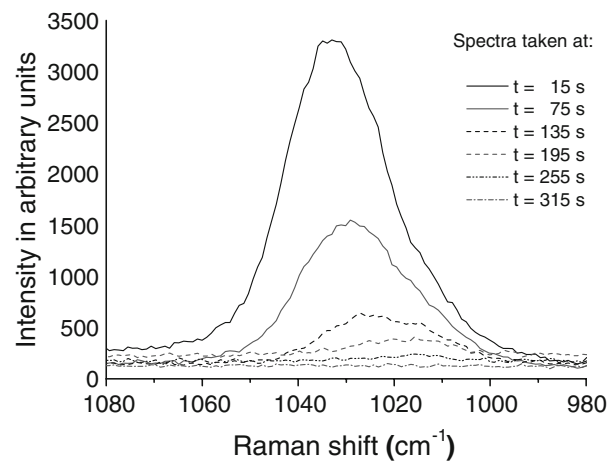

(c)

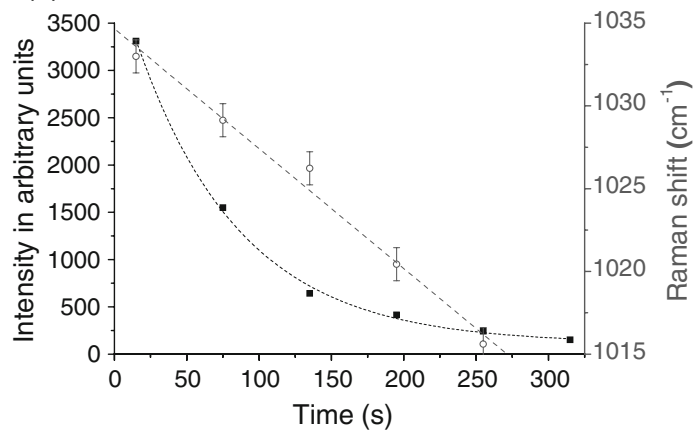

between the two extreme band centers, indicating the simultaneous existence of the aqueous and the crystalline state of $\left(\mathrm{NH}_{4}\right)_{2} \mathrm{SO}_{4}$.

In contrast to the case for electrodynamically levitated microdrops of $\mathrm{Na}_{2} \mathrm{SO}_{4}(\mathrm{aq})$ [27], where physical measurements of size, pressure, and temperature were used, the crystallization of an acoustically levitated drop of saturated aqueous solution of $\mathrm{Na}_{2} \mathrm{SO}_{4}$ at ambient room conditions proceeded - in the case of the results presented in Fig. 9via an intermediate state of $\mathrm{Na}_{2} \mathrm{SO}_{4} \cdot 10 \mathrm{H}_{2} \mathrm{O}$ towards anhydrous $\mathrm{Na}_{2} \mathrm{SO}_{4}$. This mechanism is indicated in Fig. 9b firstly by a change in the position of the Raman band from $982 \mathrm{~cm}^{-1}\left(\mathrm{SO}_{4}{ }^{2-}\right.$ (aq)) to $989 \mathrm{~cm}^{-1}\left(\mathrm{Na}_{2} \mathrm{SO}_{4} \cdot 10\right.$ $\mathrm{H}_{2} \mathrm{O}$ ) after approximately $3 \mathrm{~min}$ and further by a second shift from 989 to $994 \mathrm{~cm}^{-1}\left(\mathrm{Na}_{2} \mathrm{SO}_{4}\right)$ after approximately
$21 \mathrm{~min}$. The band positions for the three species are assigned through good agreement with the values of Fung and Tang [25]. In the interval between 3 and $20 \mathrm{~min}$ (labeled by B in Fig. 9a) in which the intermediate state dominated, the drop temperature has been continuously increased while it dropped down again when recrystallization towards anhydrous $\mathrm{Na}_{2} \mathrm{SO}_{4}$ started.

Acid-base microtitration inside acoustically levitated drops

Acid-base microtitration within an acoustically levitated drop has successfully been carried out using UV-Vis spectroscopy and fluorescence spectroscopy by Rohling et al. [18] using indicators and fluorescent tags. By contrast, using Raman spectroscopy as a probe, microtitration can be
Fig. 8 Evaporation and crystallization of a saturated aqueous solution of $\left(\mathrm{NH}_{4}\right)_{2} \mathrm{SO}_{4}$ in an acoustically levitated drop in ambient air at $T=20^{\circ} \mathrm{C}$ and $\mathrm{RH}=39 \%$. The temporal evolution of drop surface area and drop surface temperatures is shown in a while Raman spectra of an evaporating and crystallizing $\left(\mathrm{NH}_{4}\right)_{2} \mathrm{SO}_{4}$ drop taken at different times (with a sampling time of $30 \mathrm{~s}$ ) during this process are shown in $\mathbf{b}$ (a)

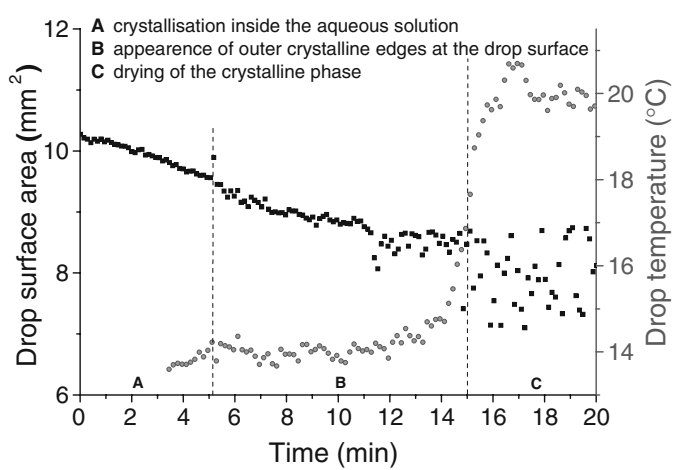

(b)

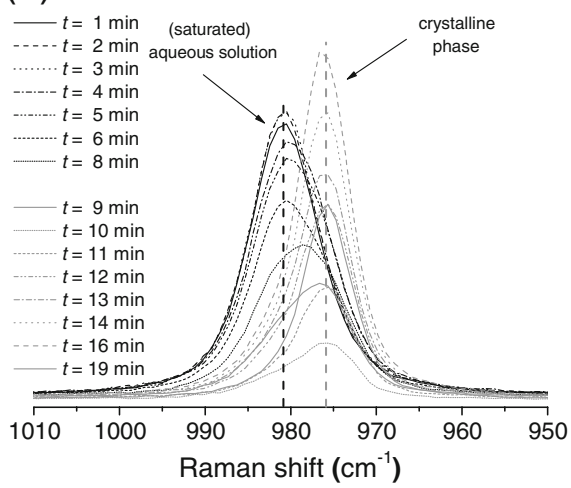


Fig. 9 Evaporation and crystallization of a saturated aqueous solution of $\mathrm{Na}_{2} \mathrm{SO}_{4}$ in an acoustically levitated drop in ambient air at $T=20^{\circ} \mathrm{C}$ and $\mathrm{RH}=42 \%$. The temporal evolution of drop surface area and drop surface temperatures is shown in a while Raman spectra of an evaporating and crystallizing $\mathrm{Na}_{2} \mathrm{SO}_{4}$ drop taken at different times (with a sampling time of $30 \mathrm{~s}$ ) during this process are shown in $\mathbf{b}$ (a)

(b)

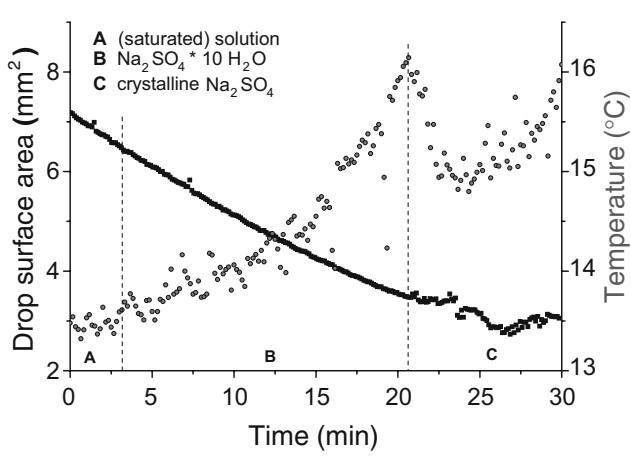

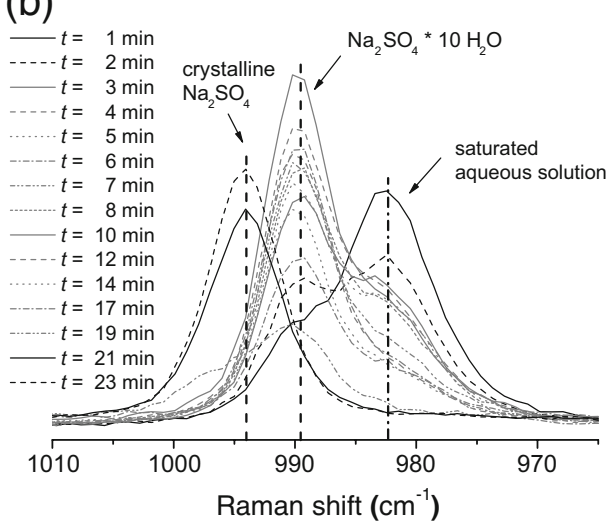

carried out without indicators by directly observing the relative intensities of single Raman bands.

Results from acid-base titrations carried out by subsequent addition of sodium hydroxide to an acoustically levitated drop of sulfuric acid are shown in Fig. 10. Solvent addition to the levitated drop was achieved using a microliter syringe with an estimated error of $\Delta V=0.1 \mu \mathrm{L}$. The intensity of the $\mathrm{SO}_{4}{ }^{2-}$ band at $982 \mathrm{~cm}^{-1}$ normalized to the intensities at $1,050 \mathrm{~cm}^{-1}$ and $982 \mathrm{~cm}^{-1}$ was used as an indicator for acid-base titration. The experimental data fit a sigmoidal curve (correlation factor $R=0.99954$ ) and the turning point $(5.015 \pm 0.085) \mu \mathrm{L}$ of the sigmoidal fit is correctly identified within the given errors of solvent addition.

\section{Conclusions}

The principles of RALS technique have been introduced and the signal intensity has been optimized with respect to the drop position. Application of the RALS technique to some simple chemical and physicochemical processes, e.g., concentration series, phase transitions, and chemical reactions, has been presented. Although conventional Raman spectroscopy is not particularly sensitive to the detection of single compounds in an acoustically levitated drop below concentrations of approximately $1 \%$, the SERS technique overcomes this limitation and the potential of RALS as a tool to investigate processes that change the molecular state and environment of the target compound is beginning to emerge. Analyte quantification using SERS however is still a difficult proposition and with our RALS apparatus in its present form the SERS signal was not consistent enough for reliable quantification. Yea et al. [28] have shown that "a highly accurate quantitative analysis is possible if a continuous flow and homogeneous mixing conditions between metals nanocolloids are maintained in a microfluidic channel" and this, combined with the automated flow through dispenser developed for RALS by Lendl et al. [9], would seem to be the most promising approach for obtaining consistent SERS spectra in levitated drops. As shown by Wood et al. [15] and Puskar et al. [14], RALS (a)

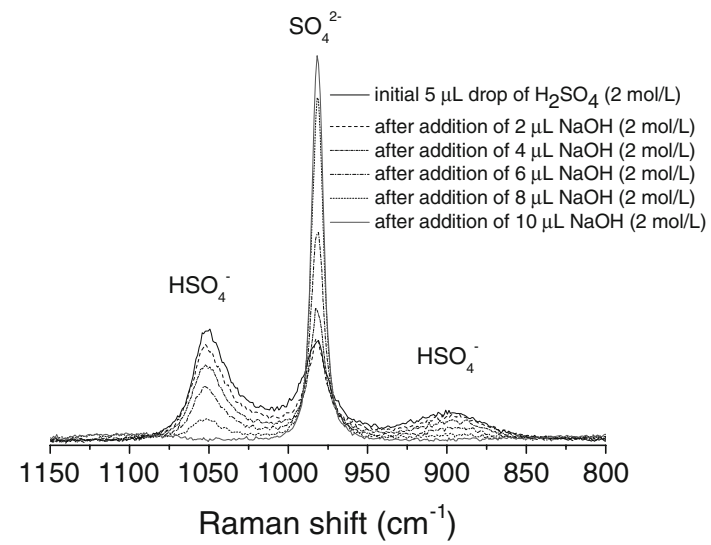

(b)

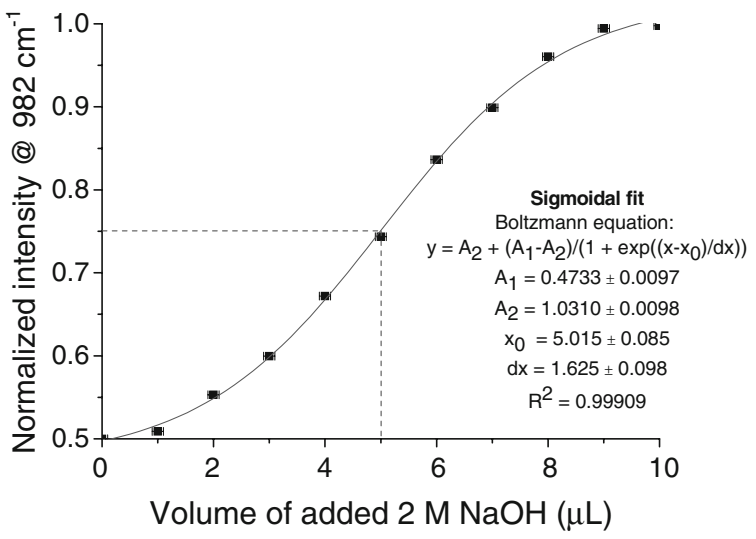

Fig. 10 Microtitration of $2 \mathrm{M}$ sulfuric acid with $2 \mathrm{M}$ sodium hydroxide: a Raman spectra (sampling time $30 \mathrm{~s})$ of the $\mathrm{SO}_{4}{ }^{2-}$ band at $982 \mathrm{~cm}^{-1}$ taken during microtitration; b titration curve 
can be also used in complex biological systems. The advantages of RALS investigation of contactless suspensions of small samples for processes highly affected by wall contacts, such as crystallization, which has relevance for example in atmospheric cloud physics and chemistry as well as in technical applications (spray drier), will be the focus of future work.

Acknowledgement The authors like to thank Finlay Shanks for general technical support. The project was funded by the Australian Research Council (ARC) and the German Research Association (DFG). RS is supported by Monash University Research scholarships and MZ by a Rural Industry Research and Development Corporation funded scholarship.

\section{References}

1. Lierke EG (1996) Acoustica 82:220-237

2. Welter E, Neidhart B (1997) Fresenius J Anal Chem 357:345-350

3. Santesson S, Nilsson S (2004) Anal Bioanal Chem 378:1704-1709

4. Priego-Capote F, de Castro L (2006) Trends Anal Chem 25:856-867

5. Petry R, Schmitt M, Popp J (2003) Chem. Phys Chem 4:14-30

6. Biswas A (1993) Appl Spectrosc 47:458

7. Biswas A (1995) J Crystal Growth 147:155-164

8. Davies AN, Jacob P, Stockhaus A, Kuckuk R, Hill W, Hergenröder R, Zybin A, Klockow D (2000) Appl Spectrosc 54:1831-1836

9. Leopold N, Haberkorn M, Laurell T, Nilsson J, Baena JR, Frank J, Lendl B (2003) Anal Chem 75:2166-2171

10. Santesson S, Johansson J, Taylor LS, Levander I, Fox S, Sepaniak M, Nilsson S (2003) Anal Chem 75:2177-2180

11. Esen C, Weigel T, Sprynchak V, Schweiger G (2004) J Quant Spectrosc Radiat Transfer 89:79-85
12. Lopez-Pastor M, Dominguez-Vidal A, Ayora-Canada MJ, Laurell T, Valcarcel M, Lendl B (2007) Lab Chip 7:126-132

13. Ruedas-Rama MJ, Dominguez-Vidal A, Radel S, Lendl B (2007) Anal Chem 79:7853-7857

14. Puskar L, Tuckermann R, Frosch T, Popp J, Ly V, McNaughton D, Wood BR (2007) Lab Chip 7:1125-1131

15. Wood BR, Heraud P, Stojkovic S, Morrison D, Beardall J, McNaughton D (2005) Anal Chem 77:4955-4961

16. Lee PC, Meisel D (1982) J. Phys Chem 86:3391-3395

17. Tuckermann R, Bauerecker S, Cammenga HK (2007) J Colloid Interface Science 310:559-569

18. Rohling O, Weitkamp C, Neidhart B (2000) Fresenius J Anal Chem 368:125-129

19. Frost RL, Kristof J, Horvath E, Kloprogge JT (1999) J Phys Chem A 103:9654-9660

20. Fleischmann M, Hendra PJ, Mcquilla AJ (1974) Chem Phys Lett 26:163-166

21. Kneipp K, Wang Y, Kneipp H, Perelman LT, Itzkan I, Dasari R, Feld MS (1997) Phys Rev Lett 78:1667-1670

22. Bell EJ, Mackle JN, Narayana MSS (2005) The Analyst 130:545-549

23. Yarin L, Brenn G, Kastner O, Rensink D, Tropea C (1999) J Fluid Mech 399:151-204

24. Tuckermann R, Bauerecker S, Cammenga HK (2005) Int J Thermophys 26:1583-1594

25. Fung KH, Tang IN (2000) Chemical characterization of aerosol particles by laser Raman spectroscopy. In: Spurny KR, Hochrainer $\mathrm{D}$ (eds) Aerosol chemical processes in the environment. CRC Press, Boca Raton

26. Trunk M, Lübben JF, Popp J, Schrader B, Kiefer W (1997) Appl Optics 36:3305-3309

27. Tang IN (2000) Phase transformation and growth of hydroscopic aerosols. In: Spurny KR, Hochrainer D (eds) Aerosol chemical processes in the environment. CRC Press, Boca Raton, p 2000

28. Yea K, Lee S, Kyong JB, Choo J, Lee EK, Joo SW, Lee S (2005) Analyst 130:1009-1011

29. Schrader B (1989) Raman/Infrared atlas of organic compounds. VCH Verlagsgesellschaft mbH, Weinheim 